\title{
Antitachycardia Pacing
}

National Cancer Institute

\section{Source}

National Cancer Institute. Antitachycardia Pacing. NCI Thesaurus. Code C101245.

Delivery of electrical impulses to the heart at a faster rate than the intrinsic rate during an episode of tachycardia, in an attempt to terminate the abnormal tachycardia. (ACC) 